\title{
Synthesis of flexible heat exchange networks and mass exchange networks
}

\author{
Cheng-Liang Chen*, Ping-Sung Hung \\ Department of Chemical Engineering National Taiwan University Taipei 10617, Taiwan, ROC \\ Received 27 January 2006; received in revised form 22 January 2007; accepted 30 January 2007 \\ Available online 9 February 2007
}

\begin{abstract}
A simple strategy is proposed for the synthesis of flexible heat exchange networks (HEN's) or mass exchange networks (MEN's) that involves expected disturbance range in the flow rates and temperatures (for HEN's) or compositions (for MEN's) of the inlet process streams. The network synthesis problem is decomposed into three main iterative steps: (1) synthesizing a network candidate with a minimum total annual cost (TAC) according to a finite number of operating conditions; (2) performing flexibility test without considering the size restrictions to verify whether the current network candidate is operable for a large number of uncertain parameters that are generated randomly within the expected operating ranges; appending test point(s) that mostly violates the constraints of the candidate network when the network configuration is proved infeasible through simulations, then returning to step (1) again to synthesize a new candidate structure; (3) considering the size restraints that have been ignored previously, executing flexibility test to the network qualified in step (2), and increasing the size of exchange units if necessary. A few iterations of these design steps may be required to secure the desirable results. Also in this paper are several numerical examples supplied to demonstrate the applicability of the proposed strategy for the synthesis of flexible heat exchange networks or mass exchange networks.
\end{abstract}

(C) 2007 Elsevier Ltd. All rights reserved.

Keywords: Heat exchange networks (HEN's); Mass exchange networks (MEN’s); Flexibility; Synthesis; Superstructure; Mixed-integer nonlinear program (MINLP)

\section{Introduction}

Heat integration is one of the most important topics for enhancing the operational efficiency in the process industries. Therein the synthesis of heat exchange networks (HEN's) is by far one of the most developed fields. Many techniques, such as the pinch design method (Linnhoff \& Hindmarsh, 1983) and the mathematical programming approaches, have been proposed for the synthesis of HEN's (Floudas, Ciric, \& Grossmann, 1986; Grossmann, Caballero, \& Yeomans, 1999; Yee, Grossmann, \& Kravanja, 1990). A thorough review for HEN synthesis can be found in one up-to-date report (Furman \& Sahinidis, 2002). Recently, the increasing needs for environmentally benign processes and waste management have motivated the consideration of mass integration as one alternative to reduce waste treatment costs. Many attempts are thus explored to extend the generally successful experience of HEN synthesis to the synthesis of mass exchange networks (MEN's, for example, see Chen \& Hung, 2005b, 2005c; El-Halwagi \& Manousiouthakis, 1989, 1990a, 1990b; Hallale \& Fraser, 2000a, 200b, 2000c, 2000d).

Heat and/or mass integration is, of course, greatly interlinked to process integration; process/heat/mass integrations typically introduce operational problems, especially regarding flexibility and controllability. The problem of including flexibility aspects at the synthesis of HEN's and/or MEN's has gradually started to receive attention over the last decade. Such issues as minimizing the utility consumption, minimizing the matching numbers, minimizing the total area for all heat/mass exchange units and achieving flexibility in a network to enable feasible operation under possible variations in source-stream flow rates and temperatures (for HEN's) and compositions (for MEN's) - all typical design objectives - have also been the subjects of some articles (for HEN's, see Floudas \& Grossmann, 1987; Grossmann \& Floudas, 1987; and for MEN's, see Zhu \& El-Halwagi, 1995). Among them, Floudas and Grossmann (1987) proposed a sequential HEN synthesis method that combines the multiperiod mixed-integer linear programming

\footnotetext{
* Corresponding author. Tel.: +8862 23636194; fax: +886223623040.

E-mail address: CCL@ ntu.edu.tw (C.-L. Chen).
} 


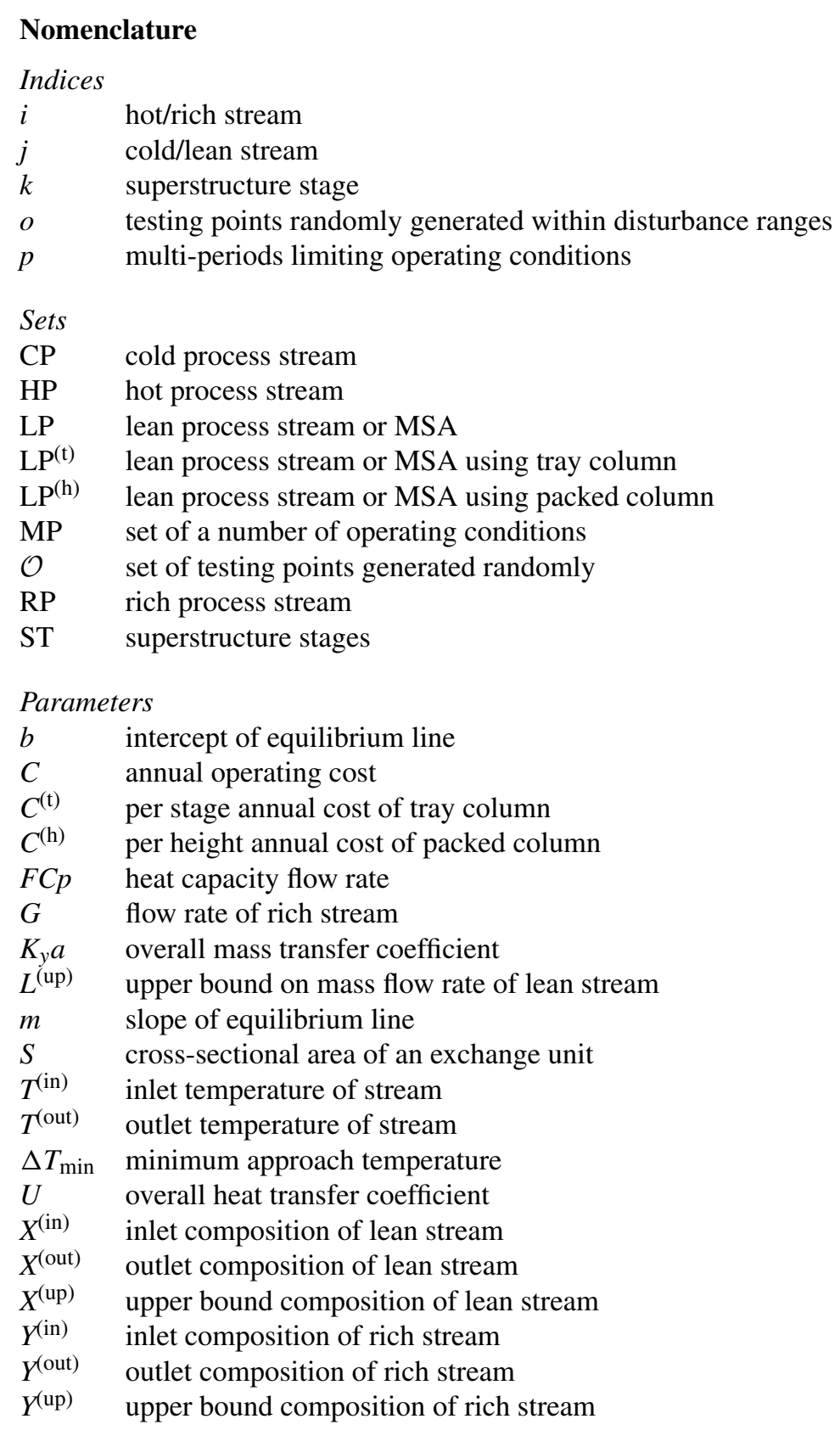

\section{Greek letters}

$\Lambda, \Gamma \quad$ large positive values

$\varepsilon_{i j} \quad$ minimum composition difference between rich stream and lean stream

$\Delta \theta^{-}, \Delta \theta^{+}$scaled deviations from $\theta^{0}$

\section{Variables}

$\mathrm{d} t_{i j k} \quad$ temperature approach for match $i$ and $j$ in stage $k$

$\mathrm{d} t c u_{i} \quad$ temperature approach for match $i$ and cold utility

$\mathrm{d} t h u_{j} \quad$ temperature approach for match $j$ and hot utility

$g_{i j k} \quad$ flow rate of rich $i$ that is connected to lean $j$ in stage $k$

$H_{i j k} \quad$ height of in packed column $(i, j, k)$

$\ell_{i j k} \quad$ flow rate of lean $j$ that is connected to rich $i$ in stage $k$

$L_{j} \quad$ flow rate of lean process stream $j$ 


\begin{tabular}{|ll|}
\hline & \\
$M_{i j k}$ & mass exchanged between rich stream $i$ and lean stream $j$ in stage $k$ \\
$N s t_{i j k}$ & number of trays in tray column $(i, j, k)$ \\
$q_{i j k}$ & heat exchanged between streams $i$ and $j$ in stage $k$ \\
$q c u_{i}$ & heat exchanged between stream $i$ and cold utility \\
$q h u_{j}$ & heat exchanged between stream $i$ and hot utility \\
$s x_{i j k}$ & composition of the part of lean $j$ that is connected to rich $i$ in rich end of an exchanger in stage $k$ \\
$s y_{i j k}$ & composition of the part of rich $i$ that is connected to lean $j$ in lean end of an exchanger in stage $k$ \\
$t_{i k}$ & temperature of stream $i$ at hot end of stage $k$ \\
$t_{j k}$ & temperature of stream $j$ at hot end of stage $k$ \\
$x_{j k}$ & composition of lean stream $j$ in rich end of stage $k$ \\
$y_{i k}$ & composition of rich stream $i$ in rich end of stage $k$ \\
$z_{i j k}$ & binary variable for existence of unit for match $i$ and $j$ in stage $k$ \\
$z c u_{i}$ & binary variable for existence of unit for match $i$ and cold utility in stage $k$ \\
$z h u_{i}$ & binary variable for existence of unit for match $j$ and hot utility in stage $k$ \\
\hline
\end{tabular}

(MILP) transshipment model with the active set strategy to guarantee the desired HEN flexibility. Papalexandri and Pistikopoulos (1994) proposed a multiperiod mixed-integer nonlinear programming (MINLP) model for the simultaneous synthesis of flexible heat and mass exchanger networks. Recently, Konukman, Camurdan, and Akman (2002) presented a MILP model, Aaltola (2002) proposed using a multi-period simultaneous MINLP model to generate flexible HEN's directly. Chen and Hung (2004, 2005a) combined the advantages of both approaches to synthesize flexible HEN's. Zhu and El-Halwagi (1995) proposed a new algorithm for the synthesis of flexible MEN's that can accommodate a given range of potential variations in the input streams. However, these formulations for synthesis of flexible HEN's or MEN's are somewhat intricate for many designers.

In this paper, the synthesis problem for flexible HEN's or MEN's is decomposed into iterative decision steps. In the simultaneous synthesis step, the problem is formulated as a MINLP problem for minimizing the TAC of the network, based on the modified stage-wise superstructure proposed originally by Yee et al. (1990). Instead of applying the tedious mathematical analysis in the flexibility test step, we solve the flexibility index evaluation problem for the network structure by directly applying a lot of simulations where the various input conditions are generated randomly within the possible operational ranges (Aaltola, 2002, 2003). The restriction on the finite units size is ignored temporarily to simplify the flexibility analysis. When the resulting network candidate does not pass the simplified flexibility test, the most violating point(s) can be taken into account as the additional period to exclude the same network from further synthesis. The units size of the qualified network is finally adjusted if necessary to guarantee flexible operation over the whole expected disturbance range. One major advantage of proposed strategy for synthesis of flexible HEN's or MEN's is that no tedious mathematical feasibility analysis is needed. To obtain the final qualified network configuration, several iterations of network synthesis, flexibility analysis and expansion of units' sizes are usually required. Two numerical examples are provided to demonstrate the efficiency of the proposed strategy for the synthesis of flexible HEN's or MEN's.

\section{Proposed strategy for flexible HEN or MEN synthesis}

A HEN (or MEN) synthesis problem with $N_{\mathrm{H}}$ hot $\left(N_{\mathrm{R}}\right.$ rich) streams and $N_{\mathrm{C}}$ cold $\left(N_{\mathrm{L}}\right.$ lean) streams along with possible variations in input temperatures (compositions) and flow rates is studied here. Let $\theta$ denote an uncertain parametric vector (e.g., input temperatures/compositions and flow rates) with a nominal value $\theta^{0}$ and the expected deviations of uncertain parameters in the negative and positive directions denoted by $\Delta \theta^{-}$and $\Delta \theta^{+}$, respectively. The flexible HEN (MEN) synthesis problem can then be defined as that of synthesizing a cost-effective HEN (MEN) that is workable for all possible operating points contained in $P(\delta=1)($ Floudas $\&$ Grossmann, 1987), where

$$
P(\delta)=\left\{\theta \mid \theta^{0}-\delta \Delta \theta^{-} \leq \theta \leq \theta^{0}+\delta \Delta \theta^{+}\right\}
$$

As pointed out in the literature (Grossmann, Halemane, \& Swaney, 1983; Marselle, Morari, \& Rudd, 1982; Swaney \& Grossmann, 1985), this problem is difficult to solve directly because the feasible region defined by the inequality constraints is nonconvex (Floudas, 1995), so the critical point that confines the solution might not rest on the vertices of the polyhedral region of uncertainty. It is this difficulty that motivates us to divide the flexible HEN (MEN) design into iterative steps, as depicted in Fig. 1. Instead of synthesizing the flexible HEN's or MEN's by directly solving the tedious mathematical problems, the proposed strategy involves the following iterative steps: 


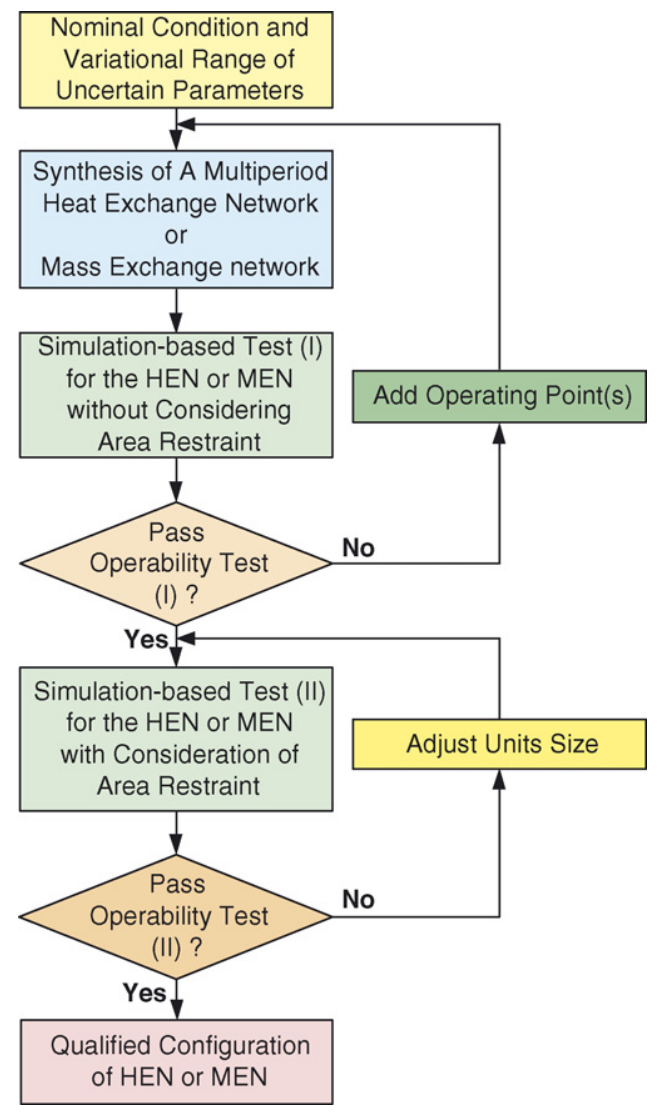

Fig. 1. Proposed strategy for synthesis of a flexible HEN or MEN.

\subsection{Step 1: Synthesis of a multiperiod network via simultaneous optimization}

According to the superstructure-based multiperiod MINLP formulation for HEN (or MEN) synthesis discussed in Section 3, a heat (or mass) exchange network can be synthesized by means of any existing mixed-integer nonlinear optimization algorithm. Several possible operating periods can be considered simultaneously to reduce the search space and to speed up the convergence, although the number of continuous variables will be increased dramatically. It is noted that the flexibility requirement is not directly taken into account in this synthesis step in order to simplify the computational load.

\subsection{Step 2: Flexibility test without considering restraints on unit sizes}

Without considering the limitation imposed on the unit sizes, we use a lot of testing conditions generated randomly within the possible operating ranges to examine the flexibility of the network resulting from previous synthesis step. Such simulation tests can determine, though not guarantee, whether the current network satisfies the assigned flexibility target, that is, whether the network can be operated over the full range of possible variations in assigned input streams. Here, the flexibility is determined by using the random points simulation in order to take into account the possibility of nonextreme critical points. If the resulting flexibility does not satisfy the target, then a new network candidate with improved operational flexibility must be found. To guarantee obtaining a different network structure, the point that mostly violates the simplified flexibility test can be taken into account simultaneously in the synthesis of new network candidate for speeding up convergence. On the other hand, if the current network structure conforms its flexibility to the requirement of the flexibility target, it can be temporarily accepted as a qualified network configuration to facilitate further examination with the consideration of size constraints, such as followed by step 3 .

\subsection{Step 3: Re-sizing of exchangers}

For a network structure that has been qualified in the previous simplified flexibility test, some of the exchange units could be re-sized if necessary to guarantee flexible operation over the whole disturbance range.

Several iterations of design steps 1 and 2 are sometimes required to obtain a qualified configuration. To illustrate how the whole synthesis procedure can be performed, two examples for synthesis of a flexible HEN and a flexible MEN, respectively, will be 


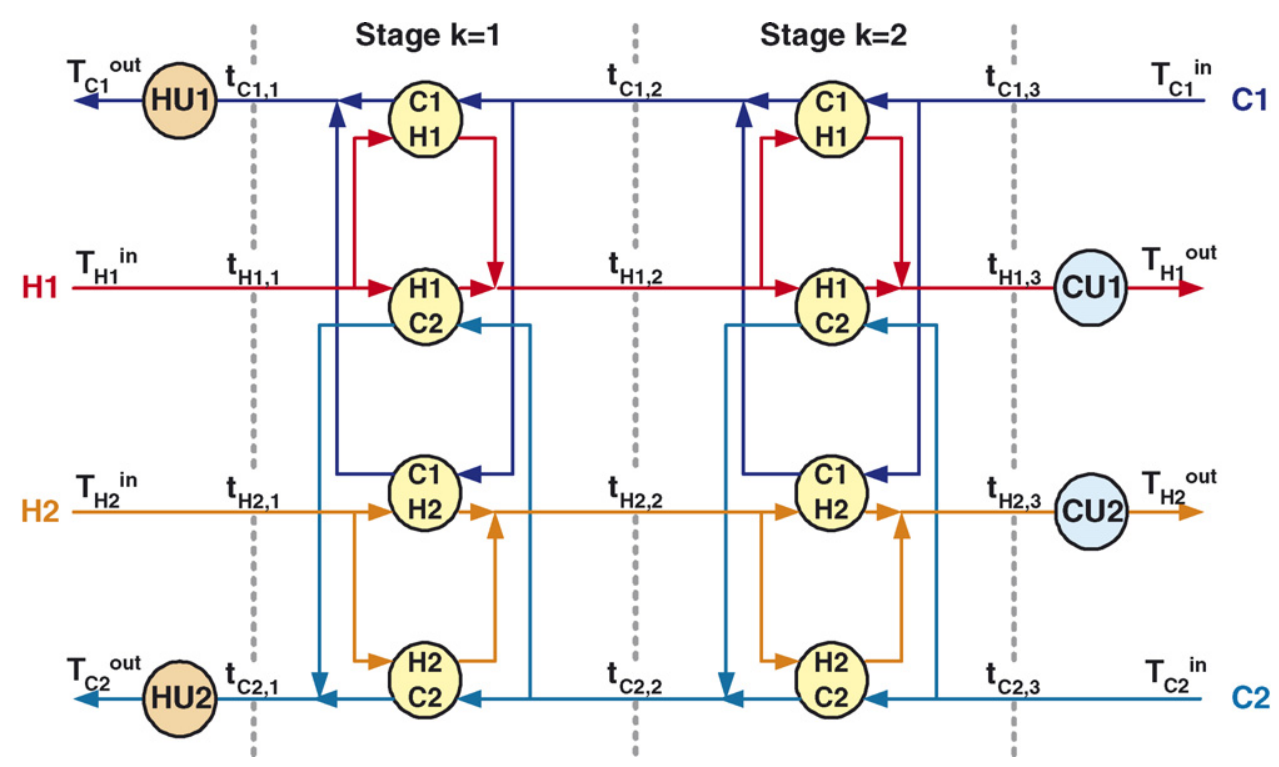

Fig. 2. The illustrative two-stage superstructure with two-hot and two-cold streams for HEN synthesis.

provided in Section 5. Through numerical simulations, it can be verified that the proposed strategy is easy to implement and can provide a feasible and balanced solution for heat exchange network or mass exchange network synthesis problems.

\section{Synthesis of multiperiod heat exchange networks or mass exchange networks}

\subsection{Heat exchange network synthesis (HENS)}

The stage-wise superstructure originally proposed by Yee et al. (1990) is applied to construct the network structure, for its suitability for formulating the simultaneous solution that involves the consideration of total utility consumption, the total number of matches and the total area of heat exchange units. A two-stage superstructure with two-hot and two-cold streams is illustrated in Fig. 2; the meanings of relevant notations of the structure can be found in nomenclature.

Instead of directly considering all possible combinations of uncertain input temperatures and heat capacity flow rates for the flexible network synthesis, only a finite number of possible operating conditions are taken into account for network design to reduce the searching space. The mathematical programming formulation for minimizing the total annual cost, TAC, which includes the average costs of the hot and cold utility consumptions over a finite number of operating points and the annual costs of installation and material of the heat exchange units, can be summarized as follows (Biegler, Grossmann, \& Westerberg, 1997; Chen \& Hung, 2005a; Yee et al., 1990):

$$
\begin{aligned}
\min _{x_{H} \in \Omega_{H}} \mathrm{TAC}= & \frac{1}{N_{p}+1}\left(\sum_{\forall p \in \mathrm{MP} \forall i \in \mathrm{HP}} \sum_{c u} q c u_{i}^{p}+\sum_{\forall p \in \mathrm{MP} \forall j \in \mathrm{CP}} C_{h u} q h u_{j}^{p}\right) \\
& +\sum_{\forall i \in \mathrm{HP} \forall j \in \mathrm{CP} \forall k \in \mathrm{ST}} \sum_{i j} C_{i j k}+\sum_{\forall i \in \mathrm{HP}} C_{i, c u} A_{i, c u}^{\beta_{i, c u}}+\sum_{\forall j \in \mathrm{CP}} C_{h u, j} A_{h u, j}^{\beta_{h u, j}}
\end{aligned}
$$

where $x_{H}$ and $\Omega_{H}$ denote the design variables and the feasible searching space comprising all material/energy balance constraints and relevant logical constraints. $\mathrm{MP}=\left\{0,1, \ldots, N_{p}\right\}$ is the index set, in which zero denotes the nominal condition (or the base case), for numbering a limited number of operating points used for finding the optimal final structure. These possible operating conditions are applied here to help reduce the searching space in the design stage.

$$
x_{H} \equiv\left\{\begin{array}{r}
z_{i j k}, z c u_{i}, z h u_{j} ; \quad A_{i j k}, A_{i, c u}, A_{h u, j} ; \quad q_{i j k}^{p}, q c u_{i}^{p}, q h u_{j}^{p} \\
t_{i k}^{p}, t_{j k}^{p}, t_{i, k+1}^{p}, t_{j, k+1}^{p} ; \quad \mathrm{d} t_{i j k}^{p}, \mathrm{~d} t_{i j, k+1}^{p}, \mathrm{~d} t c u_{i}^{p}, \mathrm{~d} t h u_{j}^{p} \\
\forall i \in \mathrm{HP}, j \in \mathrm{CP}, k \in \mathrm{ST}, p \in \mathrm{MP}
\end{array}\right\}
$$




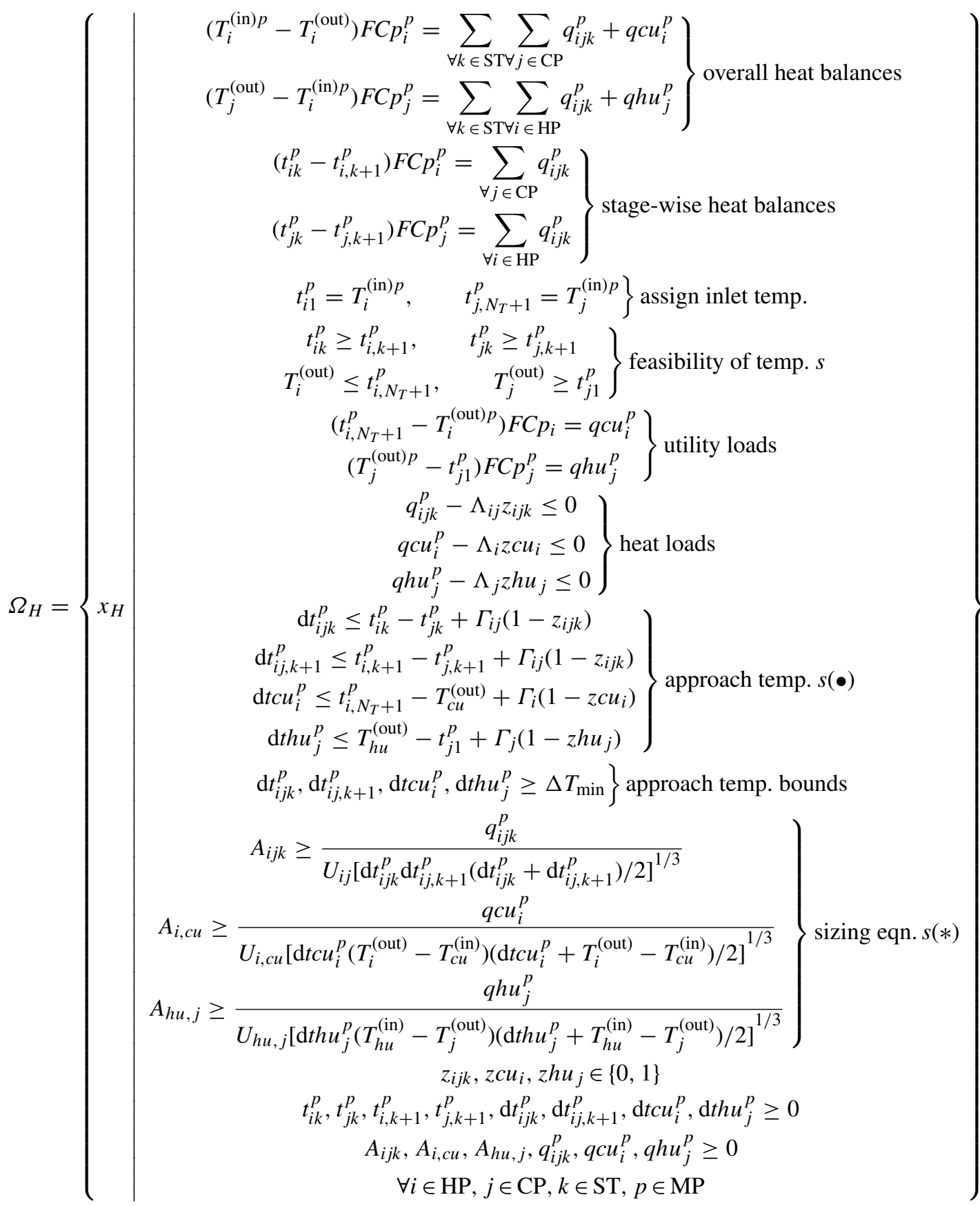

\subsection{Mass exchange network synthesis (MENS)}

The stage-wise superstructure for MEN's is applied to construct the network structure, for it is also suitable for formulation of the simultaneous solution that involves the consideration of the total consumption of mass separating agents, the total number of stream matches and the total size of mass exchange units (Chen \& Hung, 2005b, 2005c; Szitkai et al., 2003). A two-stage superstructure with two-rich and two-lean streams is shown in Fig. 3 for illustration; the meanings of relevant notations of the structure can be found in nomenclature.

A finite number of operating points are simultaneously taken into account for MEN synthesis. The mathematical programming formulation to minimize the total annual cost, TAC, which includes the average costs of external mass separating agents over a finite number of operating points and the annual costs of installation and material of the mass exchange units, can be summarized as follows, where $x_{M}$ and $\Omega_{M}$ denote the design variables and the feasible searching space, respectively, for synthesis of mass exchange 
networks (Chen \& Hung, 2005b, 2005c):

$$
\begin{aligned}
& \min _{x_{M} \in \Omega_{M}} \mathrm{TAC}=\frac{1}{N_{p}+1} \sum_{j \in \mathrm{LP}} C_{j} L_{j}^{p}+\sum_{\forall i \in \mathrm{RP} \forall j \in \mathrm{LP}(t) \forall k \in \mathrm{ST}} \sum_{i j} C_{(t)}^{(t)} N s t_{i j k}+\sum_{\forall i \in \mathrm{RP} \forall j \in \mathrm{LP}^{(h)} \forall k \in \mathrm{ST}} \sum_{i j} C_{i j k}^{(h)} H_{i j k} \\
& x_{M} \equiv\left\{\begin{array}{r}
z_{i j k}, N s t_{i j k}, H_{i j k} ; \quad L_{j}^{p}, g_{i j k}^{p}, \ell_{i j k}^{p}, M_{i j k}^{p} \\
y_{i k}^{p}, x_{j k}^{p}, y_{i, k+1}^{p}, x_{j, k+1}^{p}, s y_{i j k}^{p}, s x_{i j k}^{p} ; \quad \mathrm{d} y x i_{i j k}^{p}, \mathrm{~d} y x o_{i j k}^{p} \\
\forall i \in \mathrm{RP}, j \in \mathrm{LP} / \mathrm{LP}^{(t)} / \mathrm{LP}^{(h)}, k \in \mathrm{ST}, p \in \mathrm{MP}
\end{array}\right\}
\end{aligned}
$$

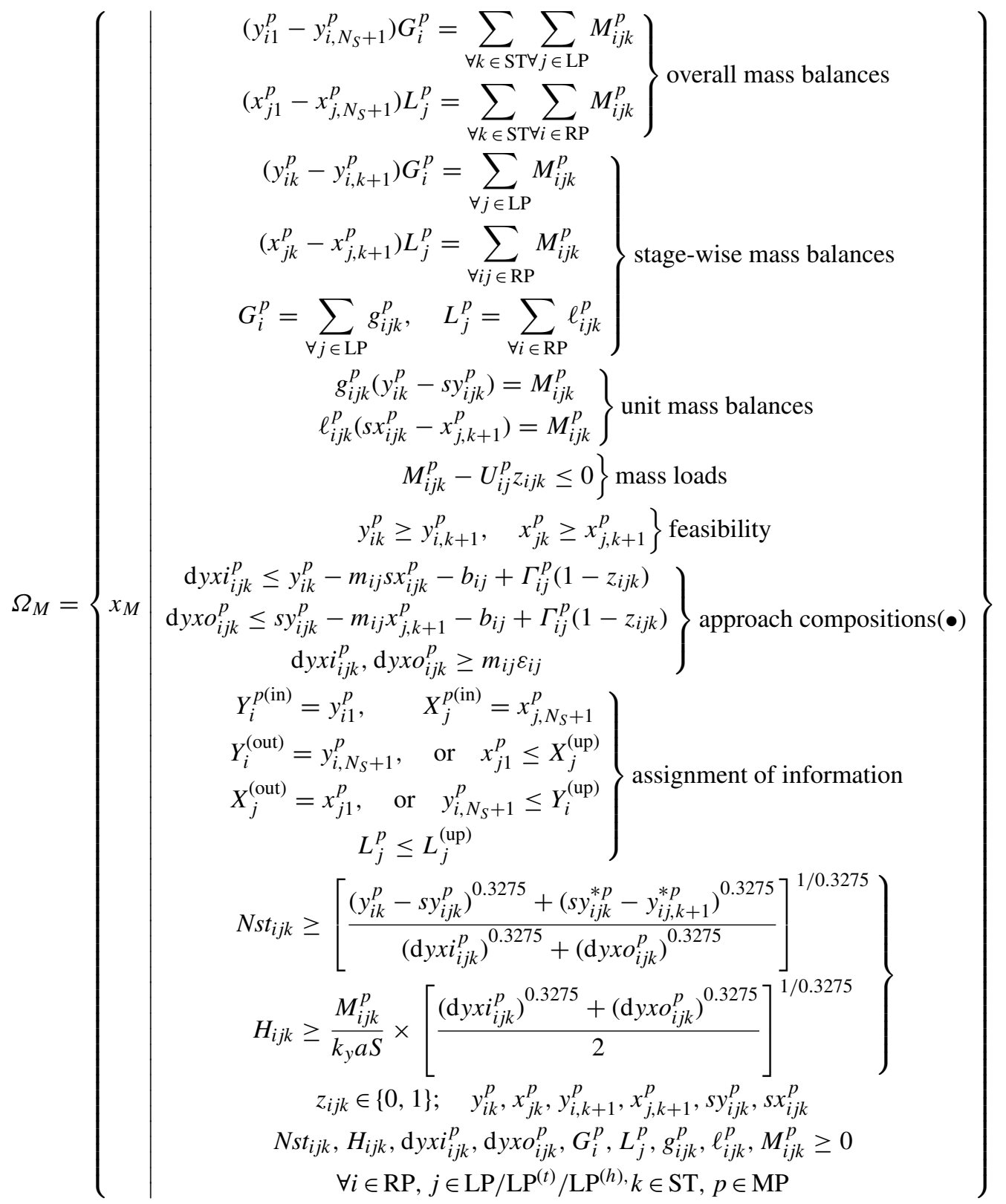

\section{Simulation-based flexibility test}

Procedures for flexibility tests for HEN's or MEN's with/without considering the restraints of units sizes are quite similar. We depict the testing steps for HEN's at first and the similarities and the differences for MEN's are then following. 


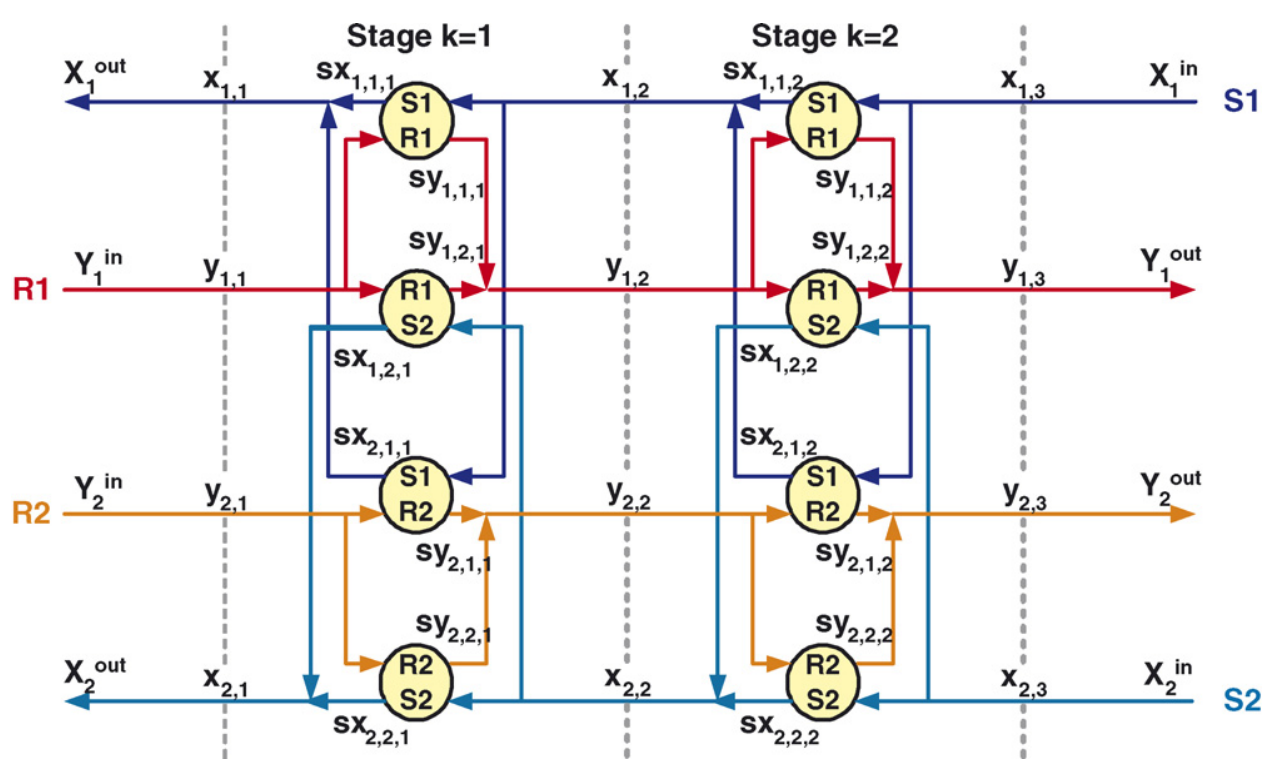

Fig. 3. The illustrative two-stage superstructure with two-rich and two-lean streams for MEN synthesis.

\subsection{Flexibility test for heat exchange networks}

There are two stages in the proposed simulation-based flexibility tests. The restrictions concerned with the unit sizes are ignored at the first testing stage to reduce the computation load. Based on the qualified network structure, one can adjust the unit sizes in the second testing step for considering all restraints. The details are given below.

\subsubsection{Flexibility test ignoring units area constraints}

According to the resulting multi-period heat exchange network configuration from Section 3, the calculation of temperature differences around all matched units in each period are examined to ensure feasible driving force for all units. We apply binary parameters $z_{i j k}^{(\mathrm{ex})}, z c u_{i}^{(\mathrm{ex})}$ and $z h u_{j}^{(\mathrm{ex})}$ to denote the existence of units in the superstructure for current network when executing the simplified flexibility analysis. That is, the $z_{i j k}^{(\mathrm{ex})}$ value is assigned to be one when the $(i, j)$ match at stage $k$ is existing, and the value is zero otherwise. The relations concerned with approach temperatures are, respectively, multiplied with the given binary parameters $z_{i j k}^{(\mathrm{ex})}, z c u_{i}^{(\mathrm{ex})}$ and $z h u_{j}^{(\mathrm{ex})}$ for relaxing the equality constraints for those units that are not existent in the candidate network. A lot of testing points $o \in \mathcal{O}$ randomly distributed within the uncertain parametric domain are then applied to examine if the current network structure can be accepted. For those existing units, slack variables, $s \mathrm{~d} t_{i j k}^{o}, s \mathrm{~d} t c u_{i j k}^{o}$ or $s \mathrm{~d} t h u_{i j k}^{o}$, are used for measuring the violation of approach temperatures (Aaltola, 2003), where the units area are not considered temporarily to simplify the flexibility test.

$$
\begin{aligned}
& \left.z_{i j k}^{(\mathrm{ex})}\left(\mathrm{d} t_{i j k}^{o}-\left(t_{j k}^{o}-t_{j k}^{o}+s \mathrm{~d} t_{i j k}^{o}\right)\right)=0\right) \quad i \in \mathrm{HP}, \\
& z_{i j, k+1}^{(\mathrm{ex})}\left(\mathrm{d} t_{i j, k+1}^{o}-\left(t_{i, k+1}^{o}-t_{j, k+1}^{o}+s \mathrm{~d} t_{i j, k+1}^{o}\right)\right)=0 \quad j \in \mathrm{CP}, \\
& \left.z c u_{i}^{\text {(ex) }}\left(\mathrm{d} t c u_{i}^{o}-\left(t_{i, N_{T}+1}^{o}-T_{c u}^{o \text { (out) }}+s \mathrm{~d} t c u_{i}^{o}\right)\right)=0\right\} k \in \mathrm{ST}, \\
& z h u_{j}^{(\mathrm{ex})}\left(\mathrm{d} t h u_{j}^{o}-\left(T_{h u}^{o(\text { out })}-t_{j, 1}^{o}+s \mathrm{~d} t h u_{j}^{o}\right)\right)=0 \quad o \in \mathcal{O}
\end{aligned}
$$

After this, the simplified flexibility testing problem can be written as the following linear program (LP) to minimize the summation of these slack variables for each testing point.

$$
\min _{x_{H^{\prime}} \in \Omega_{H^{\prime}}} J_{H^{\prime}}^{o}=\sum_{\forall i \in \mathrm{HP} \forall j \in \mathrm{CP} \forall k \in \mathrm{ST}} \sum_{i j k}\left(z_{i \mathrm{ex})} s \mathrm{~d} t_{i j k}^{o}+z_{i j, k+1}^{(\mathrm{ex})} s \mathrm{~d} t_{i j, k+1}^{o}\right)+\sum_{\forall i \in \mathrm{HP}} z c u_{i}^{(\mathrm{ex})} s \mathrm{~d} t c u_{i}^{o}+\sum_{\forall j \in \mathrm{CP}} z h u_{j}^{(\mathrm{ex})} s \mathrm{~d} t h u_{j}^{o}, \quad \forall o \in \mathcal{O}
$$

where the design variables $x_{H^{\prime}}$ are similar to $x_{H}$ with new slack variables and assigned values for all binary variables. $\Omega_{H^{\prime}}$ is equivalent to $\Omega_{H}$ with all periods $p \in$ MP being replaced by testing points $o \in \mathcal{O}$, those equations marked with $\bullet$ in Eq. (4) being replaced by Eq. (8), and unit area-dependent constraints (marked with ${ }^{*}$ ) being ignored temporarily. The idea of this formulation is to minimize the overall violation of temperature approaches for each set of testing data. The most violating point, i.e., the testing point with maximum positive $J_{H^{\prime}}^{o}$, value over all $o \in \mathcal{O}$, can be taken as an additional period for subsequent network synthesis. That is, when at lease one testing point fails to pass the simplified LP flexibility test, then the multi-periods MINLP model is resolved for 
network synthesis with this additional period included. The loop, including the MINLP synthesis step and the LP flexibility test as shown in Fig. 1, should be repeated until the resulting network is feasible for all test points in the whole specified range of parametric variations.

\subsubsection{Flexibility test considering restraints on units area}

For those network structure accepted by previous LP flexibility test, the units area can be expanded if necessary. Let $s a_{i j k}, s a_{i, c u}$ and $s a_{h u, j}$ be the slack variables which denote the additional area required for process heat exchangers, coolers and heaters, respectively (Aaltola, 2003). The required units area can be represented by the following additional constraints.

$$
\begin{aligned}
& H A_{i j k}+s a_{i j k} \geq \frac{q_{i j k}^{o}}{U_{i j}\left[\mathrm{~d} t_{i j k}^{p} \mathrm{~d} t_{i j, k+1}^{o}\left(\mathrm{~d} t_{i j k}^{o}+\mathrm{d} t_{i j, k+1}^{o}\right) / 2\right]^{1 / 3}}
\end{aligned}
$$

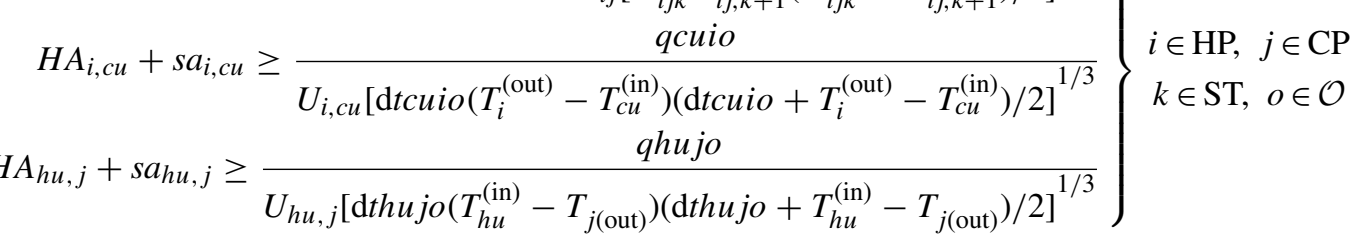

Here, $H A_{i j k}, H A_{i, c u}$ and $H A_{h u, j}$ are the current units area from previous synthesis step which are kept constant values. The objective function here is to minimize the total of additional sizes by considering a lot of testing points $o \in \mathcal{O}$. Additional area are added if the existing units sizes are not large enough for at least one testing point. The problem of finding the minimal required area can be formulated as the following nonlinear program (NLP):

$$
\min _{x_{H^{\prime \prime}} \in \Omega_{H^{\prime \prime}}} J_{H^{\prime \prime}}=\sum_{\forall i \in \mathrm{HP} \forall j \in \mathrm{CP} \forall k \in \mathrm{ST}} \sum_{\forall i \in \mathrm{HP}}\left(s a_{i j k}+s a_{i j, k+1}\right)+\sum_{\forall j \in \mathrm{CP}} s a_{i, c u}+\sum_{h u, j}
$$

In this equation, $x_{H^{\prime \prime}}$ and $\Omega_{H^{\prime \prime}}$ are design variables (including additional area, with fixed values for binary variables and existent units area) and feasible space (same as $\Omega_{H^{\prime}}$ with Eq. (10) as new constraints).

\subsection{Flexibility test for mass exchanger networks}

Similar procedures can also be applied for flexible MEN synthesis, as elucidated in the following.

\subsubsection{Flexibility test ignoring units area constraints}

To identify the feasibility of each testing point without considering the restraints on unit size, slack variables $s \mathrm{~d} y x i_{i j k}^{o}$ and $s \mathrm{~d} y x o_{i j k}^{o}$ are used to measure the violation of feasible driving forces for existing mass exchange units. The fixed binary parameters $z_{i j k}^{(\mathrm{ex})}$ are used to define whether the constraint of specific composition difference is involved or not.

$$
\begin{array}{r}
z_{i j k}^{(\mathrm{ex})}\left(\mathrm{d} y x i_{i j k}^{o}-\left(y_{i k}^{o}-m_{i j} s x_{i j k}^{o}-b_{i j}+s \mathrm{~d} y x i_{i j k}^{o}\right)\right)=0 \\
z_{i j k}^{(\mathrm{ex})}\left(\mathrm{d} y x o_{i j k}^{o}-\left(s y_{i j k}^{o}-m_{i j} x_{j, k+1}^{o}-b_{i j}+s \mathrm{~d} y x o_{i j k}^{o}\right)\right)=0 \\
\forall i \in \mathrm{RP}, j \in \mathrm{LP}, k \in \mathrm{ST}, o \in \mathcal{O}
\end{array}
$$

The problem of measuring the overall violation of each testing point $o \in \mathcal{O}$ can be formulated as the following nonlinear program.

$$
\min _{x_{M^{\prime}} \in \Omega_{M^{\prime}}} J_{M^{\prime}}^{o}=\sum_{\forall i \in \mathrm{HP} \forall j \in \mathrm{CP} \forall k \in \mathrm{ST}} \sum_{i j k} \sum_{i j k}^{(\mathrm{ex})}\left(s \mathrm{~d} y x i_{i j k}^{o}+s \mathrm{~d} y x o_{i j k}^{o}\right) \quad \forall o \in \mathcal{O}
$$

where $x_{M^{\prime}}$ and $\Omega_{M^{\prime}}$ are design variables and feasible space, respectively, which are almost the same as $x_{M}$ and $\Omega_{M}$ except that all binary variables have fixed values, all $p \in \mathrm{MP}$ are replaced by $o \in \mathcal{O}$ and Eq. (12) is included. A positive $J_{M^{\prime} \text {,min }}^{o}$ value implies the infeasible MEN structure for the oth testing point. The most violated testing point(s) can be appended as new period(s) for the synthesis of new MEN candidate. One can proceed the flexibility test considering units area restraints when all testing points have zero violation measure. 
Table 1

Cost and streams data for example 1

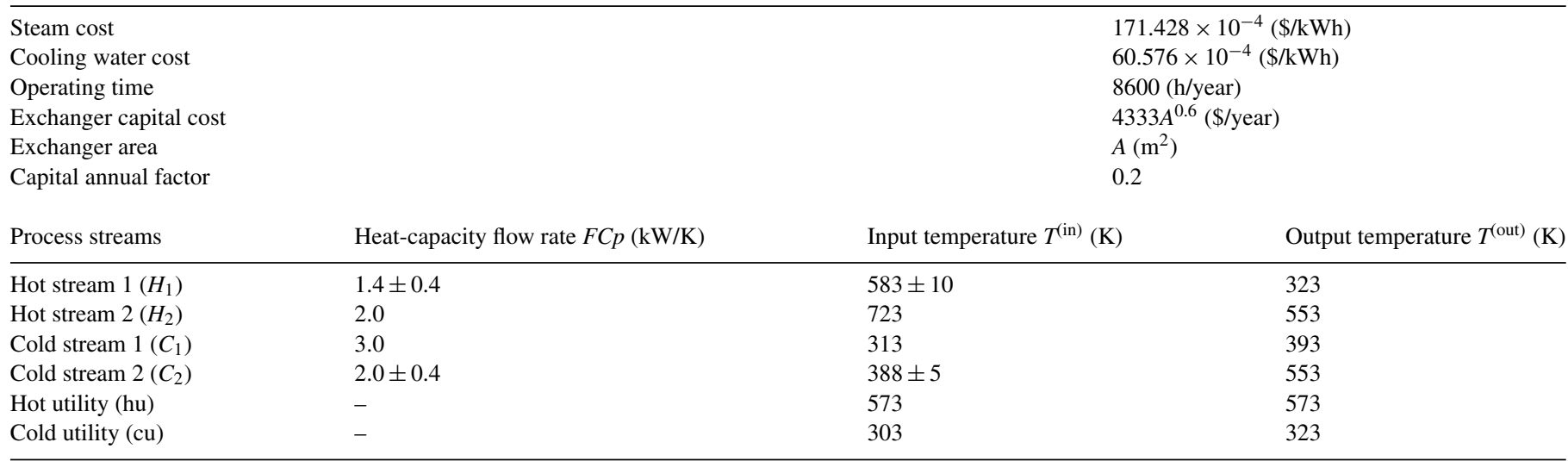

$\Delta T_{\min }=10 \mathrm{~K} . U=0.08 \mathrm{~kW} \mathrm{~m}^{-2} \mathrm{~K}^{-1}$ for all matches.

\subsubsection{Flexibility test considering units area restraints}

The similar situations for additional units sizes for MEN's can be defined.

$$
\begin{array}{r}
M N_{i j k}+s n_{i j k} \geq\left[\frac{\left(y_{i k}^{o}-s y_{i j k}^{o}\right)^{0.3275}+\left(s y_{i j k}^{o *}-y_{i j, k+1}^{o *}\right)^{0.3275}}{\left(\mathrm{~d} y x i_{i j k}^{o}\right)^{0.3275}+\left(\mathrm{d} y x o_{i j k}^{o}\right)^{0.3275}}\right]^{1 / 0.3275} \\
\forall i \in \mathrm{RP}, j \in \mathrm{LP}^{(t)}, k \in \mathrm{ST}, o \in \mathcal{O} \\
M H_{i j k}+s h_{i j k} \geq \frac{M_{i j k}^{o}}{K_{y} a S\left[\left(y_{i k}^{o}-s y_{i j k}^{o *}\right)^{0.3275}+\left(s y_{i j k}^{o}-y_{i j, k+1}^{o *}\right)^{0.3275} / 2\right]^{1 / 0.3275}} \\
\forall i \in \mathrm{RP}, j \in \mathrm{LP}^{(h)}, k \in \mathrm{ST}, o \in \mathcal{O}
\end{array}
$$

where $M N_{i j k}$ and $M H_{i j k}$ are the current tray numbers and column height, $s n_{i j k}$ and $s h_{i j k}$ are the slack variables for measuring the requirement of additional tray numbers or tower height. Slack variables are used if the exchangers sizes are not large enough when considering all testing points. The additional units size problem can then be formulated as the following nonlinear program, where the objective function is to minimize the summation of additional sizes of all units.

$$
\min _{x_{M^{\prime \prime}} \in \Omega_{M^{\prime \prime}}} J_{M^{\prime \prime}}=\sum_{\forall i \in \mathrm{HP} \forall j \in \mathrm{LP}} \sum_{(t) \forall k \in \mathrm{ST}}\left(s n_{i j k}\right)+\sum_{\forall i \in \mathrm{HP}_{\forall j}} \sum_{j \in \mathrm{LP}} \sum_{(h) \forall k \in \mathrm{ST}}\left(s h_{i j k}\right)
$$

\section{Numerical examples}

Two examples studied by Floudas and Grossmann (1987) and El-Halwagi and Manousiouthakis (1990a) will be used to demonstrate the efficiency of proposed strategy. To solve the MINLP for the related models, the General Algebraic Modeling System (GAMS) (Brooke, Kendrick, Meeraus, Raman, \& Rosenthal, 2003) is used as the main solution tool. Therein, the solvers for treating the MINLP and NLP problems are SBB and CONOPT3, respectively. The SBB solver is based on a combination of the standard Branch and Bound method known from mixed integer linear program (MILP). The CONOPT3 is well suited for models with very nonlinear constraints.

\subsection{Example 1: Synthesis of a flexible heat exchange network}

The first example involves two hot and two cold streams $\left(N_{\mathrm{H}}=2, N_{\mathrm{C}}=2\right)$ along with steam and cooling water as heating and cooling utilities. The cost and stream data of the problem are listed in Table 1. Under the condition that the expected maximum operating disturbances are $\pm 10 \mathrm{~K}$ for hot- 1 stream and $\pm 5 \mathrm{~K}$ for cold- 2 stream in temperature, and $\pm 0.4 \mathrm{~kW} \mathrm{~K}^{-1}$ for both hot- 1 and cold-2 streams in heat capacity flow rate, the objective is to derive a heat exchange network configuration that is feasible for the specified disturbance range and features the minimum TAC. The minimum number of superstructure stages, $N_{\mathrm{T}}$, is set at two because of $\max \left\{N_{\mathrm{H}}, N_{\mathrm{C}}\right\}=2$ (Yee \& Grossmann, 1990). The proposed iterative solution strategy is illustrated in the following.

(1) Iteration 1:

By applying the nominal condition to Eqs. (2)-(4), a network with a minimal TAC of US\$25,958 per year is obtained and its structure, the data of heat capacity flow rate and temperature are shown in Fig. 4, where the heat exchange load for each unit is 


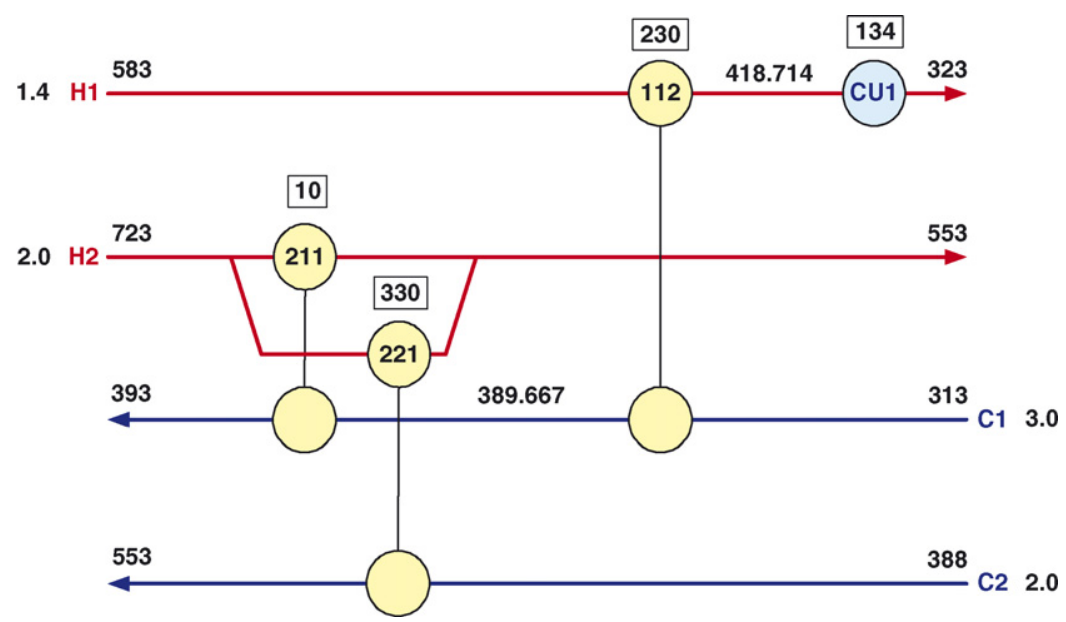

Fig. 4. HEN structure for example 1 when considering nominal condition for synthesis.

boxed above the unit sign; the heat capacity flow rate of each stream is marked along with the stream symbol; the intermediate stream temperatures are also depicted. Then 100 testing points randomly distributed within the expected disturbance ranges are generated to examine whether the candidate network is feasible without considering area restraints. The results show that there are about 50 infeasible points since they have positive $J_{H^{\prime}}^{o}$, values. This implies that the network structure designed for nominal condition is infeasible for some possible input conditions within the disturbance ranges. For example, the test point which has the largest $J_{H^{\prime}}^{o}$, value $\left(F C p_{i=1}=1.075 \mathrm{~kW} \mathrm{~K}^{-1}, F C p_{j=2}=2.180 \mathrm{~kW} \mathrm{~K}^{-1}, T_{i=1}=584.997 \mathrm{~K}, T_{j=2}=383.664 \mathrm{~K}\right)$, also named as operating point 1 , cannot be handled by the current design. Therein the heating load of cold stream $C_{2}$ is $369 \mathrm{~kW}$, which is greater than the maximum potential supply $(340 \mathrm{~kW})$ from hot stream $H_{2}$ via exchanger unit 221 . To obtain a new candidate network, operating point 1 is included for synthesis of a new network. That is, the new heat exchange network will be synthesized in the next iteration to satisfy both nominal condition and operating point 1 simultaneously.

(2) Iteration 2:

With additional operating point 1 for synthesizing a new network candidate, Eqs. (2)-(4) are solved again. The resulting network, shown in Fig. 5, features a TAC of US\$30,126 per year. One hundred test points are used to examine whether the new candidate is feasible or not. The results show that four test points are still infeasible. This implies that the second network structure designed for the nominal condition and operating point 1 is still infeasible for some possible input conditions within the disturbance ranges. Therein, the test point, which has the largest $J_{H^{\prime}}^{o}$ value $\left(F C p_{i=1}=1.157 \mathrm{~kW} \mathrm{~K}^{-1}, F C p_{j=2}=2.383 \mathrm{~kW} \mathrm{~K}^{-1}\right.$, $T_{i=1}=575.623 \mathrm{~K}, T_{j=2}=386.421 \mathrm{~K}$ ), named as operating point 2 , will be appended to the next network synthesis problem to exclude the current network configuration again. In operating point 2, the stream cooling loads are 292.3 and $340 \mathrm{~kW}$ for $H_{1}$ and $H_{2}$, and heating loads are 240 and $397 \mathrm{~kW}$ for $C_{1}$ and $C_{2}$, respectively. Stream $C_{1}$ has to be heated by $H_{1}$ via unit 112 with a rate of $240 \mathrm{~kW}$. Therefore the maximum remain of $H_{1}$ that can be used by $C_{2}$ is $52 \mathrm{~kW}$. The remained heating load of $C_{2}, 397-52=345 \mathrm{~kW}$, is still greater than the maximal supply of $H_{2}, 340 \mathrm{~kW}$, which is infeasible. In order to obtain a better

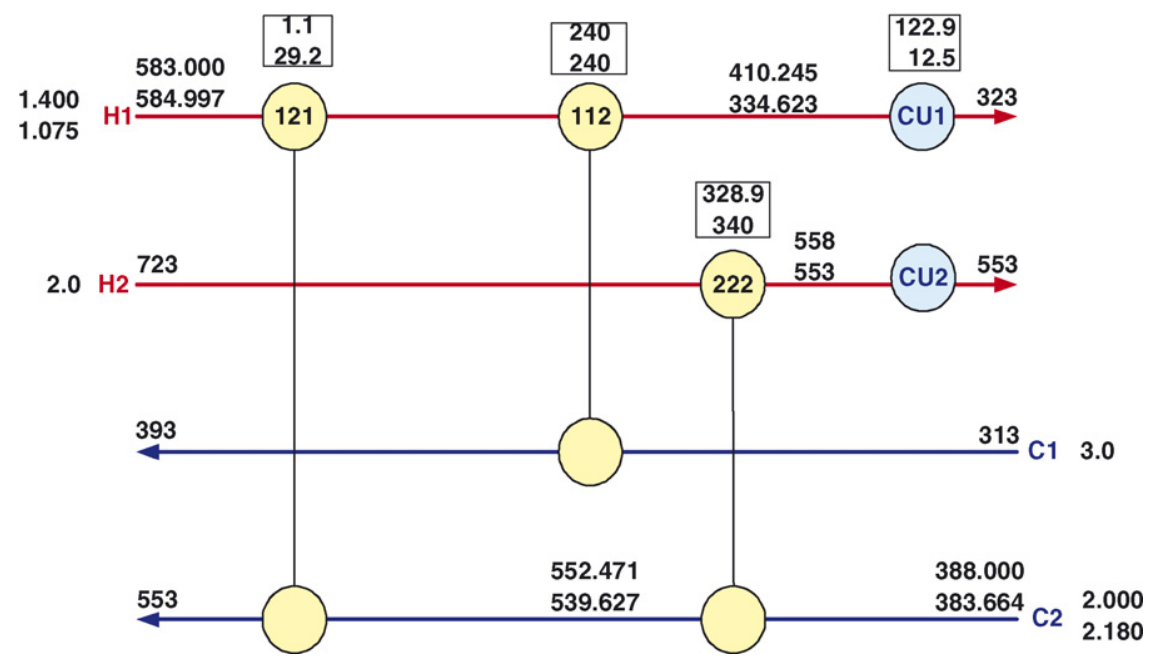

Fig. 5. HEN structure for example 1 when considering nominal condition and operating point 1 for synthesis. 


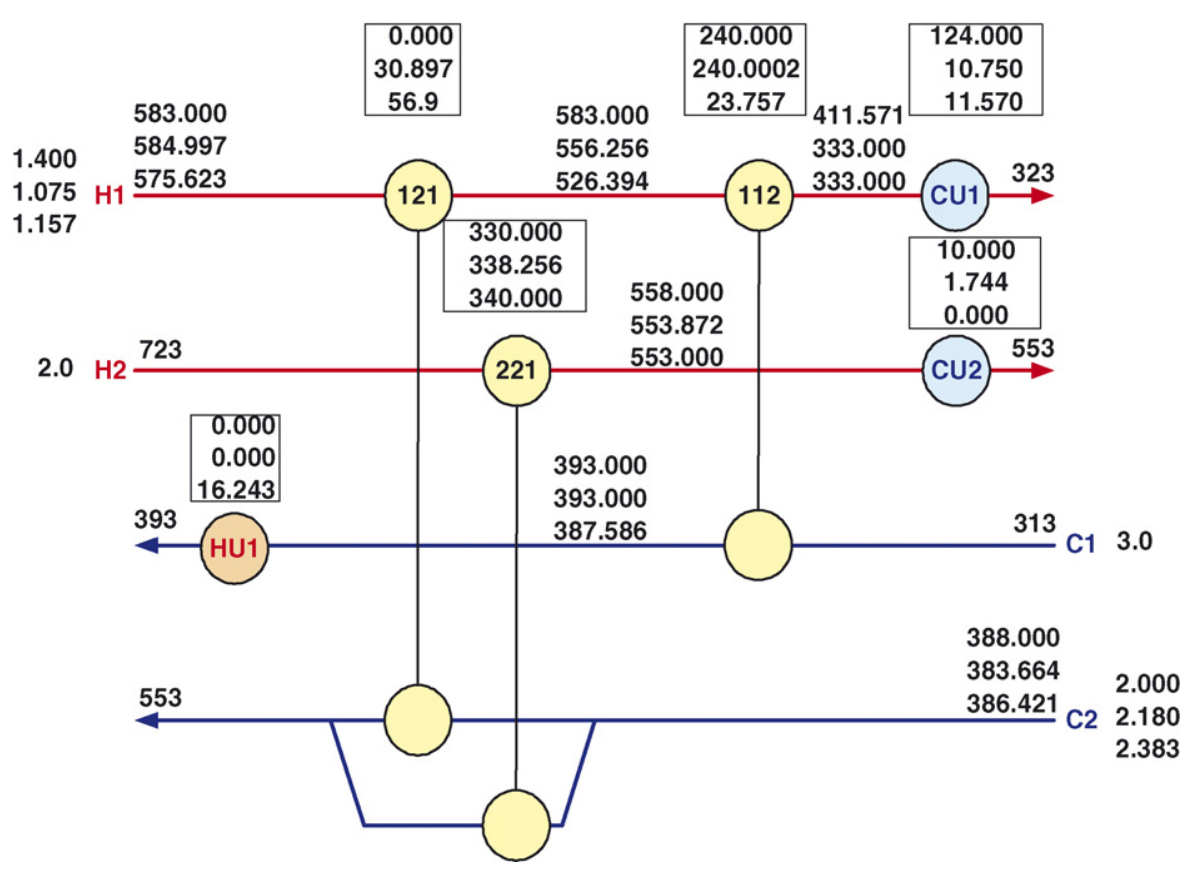

Fig. 6. HEN structure for example 1 when considering nominal condition and operating points 1 and 2 for synthesis.

network, operating point 2 is further appended to the network synthesis problem to exclude the current network configuration in the next iteration.

(3) Iteration 3:

With nominal condition and operating points 1 and 2 for excluding the previous networks obtained from iterations 1 and 2, Eqs. (2)-(4) are solved again. A new network is generated as shown in Fig. 6, which features a TAC of US\$ 32,163 per year. One hundred test points are used again to inspect flexibility of the network. The results show that all testing points are feasible. This implies that the current network structure designed for nominal condition and operating points 1 and 2 might be feasible for all possible input conditions within the disturbance ranges.

(4) Calculation of additional units area:

The flexibility test is proceeded to take the exchangers areas into account. The solution of Eq. (9) reveals that coolers 1 and 2 need additional area of 5.784 and $6.039 \mathrm{~m}^{2}$, respectively. Therefore, the TAC raises to US\$34,970 per year. The current network structure derived from third synthesis step with additional coolers area is feasible for the overall operating space.

\subsection{Example 2: Synthesis of a flexible mass exchange network}

The second example involves the removal of copper from an ammonical etching solution and a rinsewater stream. A schematic representation of the etching process can be found in El-Halwagi and Manousiouthakis (1990a). Therein the etching of copper is achieved through ammoniacal solution and the etching efficiency is higher for copper compositions between 10\% and 13\% (w/w). The copper contaminant is continuously removed from the ammoniacal solution, $R_{1}$, via solvent extraction and the regenerated etchant is returned to the etching line. Meanwhile the etched printed circuit boards are washed out with water, and the effluent rinse water, $R_{2}$ is also decontaminated by extraction and then recycled to the rinse vessel.

Two external MSAs are available for removal of copper: the LIX63 (an aliphatic $\alpha$-hydroxyoxime, $S_{1}$ ) and the so-called $P_{1}$ (an aromatic $\beta$-hydroxyoxime, $S_{2}$ ). The nominal stream data are given in Table 2 , where the ammoniacal solution, $R_{1}$, may vary in between [0.194, 0.306] kg/s for flow rate and [0.12\%, 0.14\%] w/w for composition (Zhu \& El-Halwagi, 1995). Suppose mass transfer of copper is governed by following linear equilibrium relations, where $y$ and $x$ denotes weight percent of copper in rich and lean streams, respectively (El-Halwagi \& Manousiouthakis, 1990a):

$$
\begin{array}{ll}
\left(R_{1}, S_{1}\right): & y_{1}=0.734 x_{1}+0.001 \\
\left(R_{2}, S_{1}\right): & y_{2}=0.734 x_{1}+0.001 \\
\left(R_{1}, S_{2}\right): & y_{1}=0.111 x_{2}+0.008 \\
\left(R_{2}, S_{2}\right): & y_{2}=0.148 x_{2}+0.013
\end{array}
$$

Plate-column and packed-column with $1 \mathrm{~m}$ diameter are used for $S_{1}$ and $S_{2}$, respectively. The $K_{y} a$ values are 0.685 and $0.211 \mathrm{~kg}$ copper $/ \mathrm{m}^{3} / \mathrm{s}$ for $R_{1}$ and $R_{2}$, respectively (Hallale \& Fraser, 2000d). The annualised investment costs are US $\$ 4552 N_{i j k}$ per year 
Table 2

Nominal stream data for example 2

\begin{tabular}{|c|c|c|c|c|c|}
\hline Rich streams & & $G_{i}(\mathrm{~kg} / \mathrm{s})$ & & $Y_{i}^{(\mathrm{in})}$ (mass fraction) & $Y_{i}^{\text {(out) }}$ (mass fraction) \\
\hline Ammon. soln $\left(R_{1}\right)$ & & $0.250 \pm 0.056$ & & $0.13 \pm 0.01$ & 0.10 \\
\hline Rinsewater $\left(R_{2}\right)$ & & 0.100 & & 0.06 & 0.02 \\
\hline Lean streams & $L_{j}^{\text {(up) }}(\mathrm{kg} / \mathrm{s})$ & & $X_{j}^{(\mathrm{in})}$ (mass fraction) & $X_{j}^{\text {(out) }}$ (mass fraction) & Cost $(\$ / \mathrm{kg})$ \\
\hline LIX63 $\left(S_{1}\right)$ & $\infty$ & & 0.03 & 0.07 & 0.01 \\
\hline $\mathrm{P}_{1}\left(S_{2}\right)$ & $\infty$ & & 0.001 & 0.02 & 0.12 \\
\hline
\end{tabular}

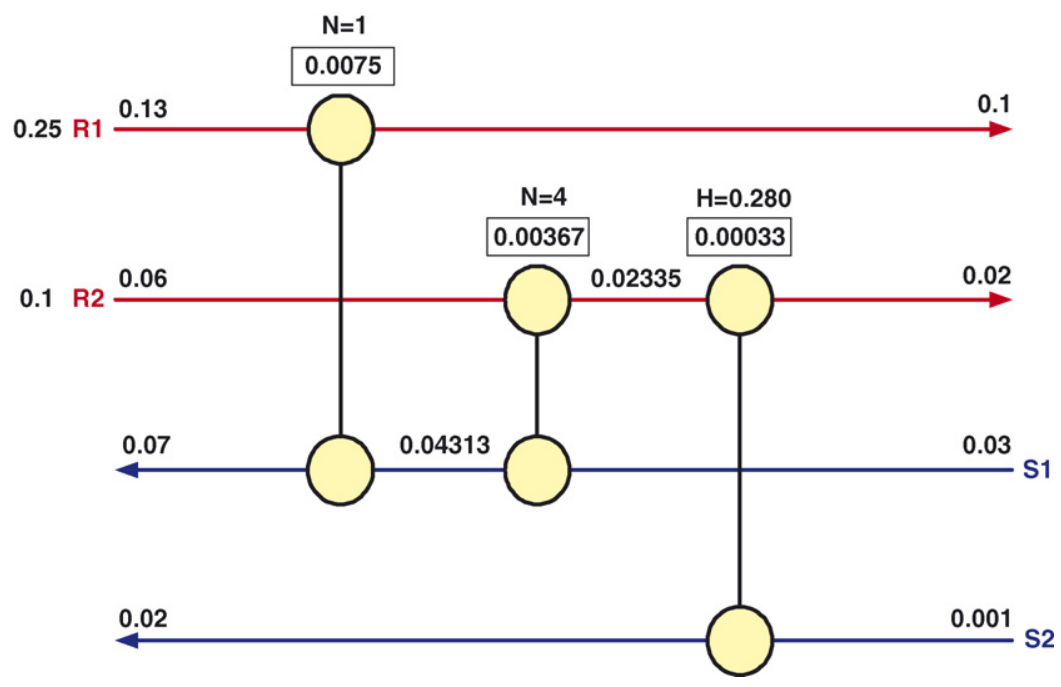

Fig. 7. MEN structure for example 2 when considering nominal condition for synthesis.

and US\$ $4245 H_{i j k}$ per year for plate-column and packed column, respectively. A minimum composition difference of $\varepsilon=0.0001$ determines feasible mass exchange at inlet and outlet of all potential mass exchange units.

(1) Iteration 1:

Firstly by applying nominal condition to Eqs. (5)-(7), a network with a minimal TAC of US\$189,359 per year is obtained and its structure and data of flow rates and compositions are shown in Fig. 7, where the mass exchange load for each unit is boxed. Then 100 test points are randomly generated within possible disturbances ranges to examine the operability of the candidate network without considering size restraints. The results show that all testing points are feasible since all $J_{M^{\prime}}^{o}$, values are zero, and this implies that the network structure designed for nominal condition only might be feasible for all possible input conditions within disturbance ranges, when the size constraints are ignored. So the current network configuration is adopted. Notice that the network configuration is the same as that designed by Papalexandri, Pistikopoulos, and Floudas (1994). The MEN reported by Zhu and El-Halwagi (1995) consists of three exchange units with a little different configuration. However, only three extreme operating points have been included in the design proposed Zhu and El-Halwagi (1995).

(2) Calculation of additional units sizes:

The flexibility test is further proceeded by including restraints on units sizes. The results show that height of the packed tower needs to increase $0.012 \mathrm{~m}$, thus the biggest violation point at $G_{1}=0.203 \mathrm{~kg} / \mathrm{s}$ and $Y_{i}^{(\mathrm{in})}=0.1205 \%(\mathrm{w} / \mathrm{w})$ will become operable. This final network is thus feasible for the overall operating space.

\section{Conclusion}

In this paper, a new simulation-based strategy for synthesis of flexible heat exchange networks or mass exchange networks that involve uncertain inlet temperatures/compositions and flow rates has been proposed. The design problem is decomposed into iterative steps: first, the stage-wise superstructure-based MINLP formulation is applied for the synthesis of a network configuration that bears the minimum total annual cost, with consideration of a finite number of operating points. Second, a large number of uncertain parameters is employed to test the operational feasibility of the candidate network. The size restraints are temporarily not considered, however, in order to simplify the computational loads. If the network structure does not satisfy the assigned flexibility 
target, then the current network is excluded by appending some operating points that are infeasible to the network constraint set, and then the search process reverts to the synthesis step to find a new candidate network. If the network passes the simplified flexibility test, then the configuration will be adopted and the flexibility test will be solved again by including the size constraints. One can make changes only in the sizes of the heat or mass exchange units in order to obtain a network that is feasible over whole expected disturbance ranges. Sometimes, several iterations are required to obtain the final qualified network. By means of two numerical examples, we have demonstrated that the proposed new strategy can generate a feasible heat or mass exchange networks for uncertain supply temperatures/compositions and flow rates in a relatively efficient way.

\section{Acknowledgements}

Financial support of Ministry of Economic Affairs under grant 94-EC-17-A-09-S1-019, and of National Science Council under grant NSC94-2214-E-002-007 are gratefully acknowledged.

\section{References}

Aaltola, J. (2002). Simultaneous synthesis of flexible heat exchanger network. Applied Thermal Engineering, 22, 907.

Aaltola, J. (2003). Simultaneous Synthesis of Flexible Heat Exchanger Networks. Ph.D. thesis. Department of Mechanical Engineering, Helsinki University of Technology.

Biegler, L. T., Grossmann, I. E., \& Westerberg, A. W. (1997). Systematic methods of chemical process design. NJ, USA: Prentice Hall, Inc..

Brooke, A., Kendrick, D., Meeraus, A., Raman, R., \& Rosenthal, R. E. (2003). GMAS: A user's guide. Redwood city: Scientific Press.

Chen, C. L., \& Hung, P. S. (2004). Simultaneous synthesis of flexible heat-exchange networks with uncertain source-stream temperatures and flow rates. I\&EC Research, 43, 5916.

Chen, C. L., \& Hung, P. S. (2005a). Multicriteria synthesis of flexible heat - exchanger networks with uncertain source - stream temperatures. Chemical Engineering and Processing, 44, 89.

Chen, C. L., \& Hung, P. S. (2005b). Simultaneous synthesis of mass exchange networks for waste minimization. Computers and Chemical Engineering, $29,1516$.

Chen, C. L., \& Hung, P. S. (2005c). Retrofit of mass-exchange networks with superstructure-based MINLP formulation. I\&EC Research, $44,7189$.

El-Halwagi, M. M., \& Manousiouthakis, V. (1989). Synthesis of mass exchange networks. AIChE Journal, 35, 1233.

El-Halwagi, M. M., \& Manousiouthakis, V. (1990a). Automatic synthesis of mass exchange networks with single component targets. Chemical Engineering Science, 45, 2813.

El-Halwagi, M. M., \& Manousiouthakis, V. (1990b). Simultaneous synthesis of mass-exchange and regeneration networks. AIChE Journal, $36,1209$.

Floudas, C. A. (1995). Nonlinear and mixed-integer optimization: Fundamentals and applications. New York: Oxford Univ. Press.

Floudas, C. A., Ciric, A. R., \& Grossmann, I. E. (1986). Automatic synthesis of optimum heat exchanger network configurations. AIChE Journal, $32,276$.

Floudas, C. A., \& Grossmann, I. E. (1987). Synthesis of flexible heat exchanger networks with uncertain flow rates and temperatures. Computers and Chemical Engineering, 11, 319.

Furman, K. C., \& Sahinidis, N. V. (2002). A critical review and annotated bibliography for heat exchanger network synthesis in the 20th century. Industrial and Engineering Chemistry Research, 41, 2335.

Grossmann, I. E., Caballero, J. A., \& Yeomans, H. (1999). Mathematical programming approaches to the synthesis of chemical process systems. Korean Journal of Chemical Engineering, 16, 407.

Grossmann, I. E., Halemane, K. P., \& Swaney, R. E. (1983). Optimization strategies for flexible chemical process. Computers and Chemical Engineering, 7, 439.

Grossmann, I. E., \& Floudas, C. A. (1987). Active constraint strategy for flexibility analysis in chemical processes. Computers and Chemical Engineering, $11,675$.

Hallale, N., \& Fraser, D. M. (2000a). Capital and total cost targets for mass exchange networks. Part 1: Simple capital cost models. Computers and Chemical Engineering, 23, 1661.

Hallale, N., \& Fraser, D. M. (2000b). Capital and total cost targets for mass exchange networks. Part 2: Detailed capital cost models. Computers and Chemical Engineering, 23, 1681.

Hallale, N., \& Fraser, D. M. (2000c). Supertargeting for mass exchange networks. Part I: Targeting and design techniques. Transactions of IChemE, 78, 202.

Hallale, N., \& Fraser, D. M. (2000d). Supertargeting for mass exchange networks. Part II: Applications. Transactions of IChemE, 78, 208.

Konukman, A. E. S., Camurdan, M. C., \& Akman, U. (2002). Simultaneous flexibility targeting and synthesis of minimum-utility heat-exchanger networks with superstructure-based MILP formulation. Chemical Engineering and Processing, 41, 501.

Linnhoff, B., \& Hindmarsh, E. (1983). The pinch design method for heat exchanger networks. Chemical Engineering Science, $38,745$.

Marselle, D. F., Morari, M., \& Rudd, D. F. (1982). Design of resilient processing plants-II: Design and control of energy management systems. Chemical Engineering Science, 37, 259.

Papalexandri, K. P., \& Pistikopoulos, E. N. (1994). A multiperiod MINLP model for the synthesis of flexible heat and mass exchange networks. Computers and Chemical Engineering, 18, 1125.

Papalexandri, K. P., Pistikopoulos, E. N., \& Floudas, C. A. (1994). Mass exchange networks for waste minimization: A simultaneous approach. Transactions of IChemE, 72, 279.

Swaney, R. E., \& Grossmann, I. E. (1985). An index for operational flexibility in chemical process design. Part I. Formulation and theory. AIChE Journal, 31 , 621.

Szitkai, Z., Farkas, T., Kravanja, Z., Lelkes, Z., Rev, E., \& Fonyo, Z. (2003). A new MINLP model for mass exchange network synthesis. In A. Kraslawski \& I. Turunen (Eds.), Proceedings ESCAPE-13, Vol. 14 (p. 323).

Yee, T. F., Grossmann, I. E., \& Kravanja, Z. (1990). Simultaneous optimization models for heat integration-I. Area and energy targeting and modeling of multi-stream exchangers. Computers and Chemical Engineering, 14, 1151.

Yee, T. F., \& Grossmann, I. E. (1990). Simultaneous optimization models for heat integration-II. Heat exchanger network synthesis. Computers and Chemical Engineering, 14, 1165.

Zhu, M., \& El-Halwagi, M. M. (1995). Synthesis of flexible mass-exchange networks. Chemical Engineering Communications, $138,193$. 